\title{
Optimal Hyper Analytic Wavelet Transform for Glaucoma Detection in Fundal Retinal Images
}

\author{
C. Raja $^{\dagger}$ and N. Gangatharan*
}

\begin{abstract}
Glaucoma is one of the most common causes of blindness which is caused by increase of fluid pressure in the eye which damages the optic nerve and eventually causing vision loss. An automated technique to diagnose glaucoma disease can reduce the physicians' effort in screening of Glaucoma in a person through the fundal retinal images. In this paper, optimal hyper analytic wavelet transform for Glaucoma detection technique from fundal retinal images is proposed. The optimal coefficients for transformation process are found out using the hybrid GSO-Cuckoo search algorithm. This technique consists of pre-processing module, optimal transformation module, feature extraction module and classification module. The implementation is carried out with MATLAB and the evaluation metrics employed are accuracy, sensitivity and specificity. Comparative analysis is carried out by comparing the hybrid GSO with the conventional GSO. The results reported in our paper show that the proposed technique has performed well and has achieved good evaluation metric values. Two 10 - fold cross validated test runs are performed, yielding an average fitness of $91.13 \%$ and $96.2 \%$ accuracy with CGD-BPN (Conjugate Gradient Descent- Back Propagation Network) and Support Vector Machines (SVM) respectively. The techniques also gives high sensitivity and specificity values. The attained high evaluation metric values show the efficiency of detecting Glaucoma by the proposed technique.
\end{abstract}

Keywords: Glaucoma, Hyper analytic transform, GSO, Cuckoo search, Support vector machines.

\section{Introduction}

The retina has been endowed with the distinctive characteristics of being the only position where blood vessels can be straightly envisaged non-invasively in vivo. The advent of highly sophisticated technology that has accelerated the growth of digital imaging mechanisms during the course of the preceding two decades modernized fundal imaging. A prospective utility of fundal digital image assessment is the skill to evaluate a huge database of fundal images in a very short span of time. There a number of consistent systems in the market for appropriate recognition of these structures in retinal photographs [7, 1]. Mechanized identification of retinal fundal images by means of digital image assessment presents gigantic prospective yields [7].

It is significant to note that Glaucoma has assumed second spot in terms of the potential reasons for the incidence of marginal loss of eyesight in individuals all over the world and it has abysmally led to the neuron deterioration of the optic nerve. As the revival of the damaged optic nerve fibres is not clinically feasible, glaucoma often escapes unnoticed in the patient still the advanced phases set in [8]. An innovative method for

$\dagger$ Corresponding Author: Department of ECE, Anjalai Ammal Mahalingam Engineering College, Kovilvenni, India. (rajachandru82@yahoo.co.in)

* Department of ECE, R.M.K. College of Engineering and Technology, Puduvoyal, India

Received: December 9, 2014; Accepted: April 13, 2015 optic nerve head fragmentation and its substantiation, in accordance with morphological functions viz. Hough transform, and an anchored active contour representation are skillfully introduced in [9]. A vigorous and computationally competent technique for the localization of the various traits and lesions in a fundus retinal image is enthusiastically offered in [10]. A lacuna in optic disc recognition is that the main blood vessels are recognized initially and then they are intersected to locate the fairly accurate position of the optic disc [11]. Tests in accordance with digital images of the retina have been extensively executed in the hospitals in the recent past; they are still devoid of vigorous mechanized support [12].

In view of the grave challenges thrown up by the function of diagnosis, modern advancements in biomedical imaging usher in efficient quantitative imaging substitutes for the recognition and administration of glaucoma. Various imaging modalities and their refinements, encompassing optical coherence tomography [13] and utilization of wavelets are high-flying methods utilized to quantitatively analyse structural and functional deformities in the eye both to monitor unevenness and to quantify the evolution of the ailment dispassionately. Retinal image assessment methods are based on computational techniques to adapt qualitative appraisals of the eye further reproducible and unbiased. Texture traits by the use of wavelet transforms (WTs) in image processing are habitually used [14] to triumph over the simplification of traits. In WT, the image 
is symbolized in terms of the frequency of content of limited regions over a variety of scales. This illustration furnishes a structure for the scrutiny of image traits, which are autonomous in dimension and can regularly be characterized by their frequency domain traits [3].

Optimal hyper analytic wavelet transform for Glaucoma detection technique from fundal retinal images is proposed in this paper. The input image features are extracted by statistical wavelet transformation. The wavelet coefficients are optimized to best fit the classifier input for the classification accuracy.

The rest of the paper is organized as follows: A brief review of researches related to the proposed technique is presented in section 2. Proposed Glaucoma detection technique is given in section 3 and the detailed experimental results and discussion are given in section 4 . The conclusion is summed up in section 5 .

\section{Literature Survey}

Many works have been carried out in literature regarding glaucoma detection using retinal images and some of them are described in this section. Jun Cheng et al.[1] shrewdly gave shape to an innovative optic disc and optic cup segmentation by means of super-pixel categorization for glaucoma tests. Kim P.Y. et al.[2] intelligently investigated the employment of fractal analysis (FA) as the foundation of a system for multiclass forecast of the evolution of glaucoma. FA was performed on pseudo 2-D images transformed from 1-D retinal nerve fiber layer data gathered from the eyes of typical individuals, and also from persons with progressive and non-progressive glaucoma. FA traits were attained by means of a boxcounting technique and a multi-fractional Brownian motion approach that integrated quality and multi-resolution analyses. Both traits were employed for Gaussian kernelbased multiclass categorization. Sensitivity, specificity, and area under receiver operating characteristic curve (AUROC) were assessed.

Dua S. et al.[3] dexterously conducted relentless research on the biased latent of wavelet traits gathered from the daubechies (db3), symlets (sym3), and biorthogonal (bio3.3, bio3.5, and bio3.7) wavelet filters. They brought to light a method to mine energy signatures attained by the use of 2-D discrete wavelet transform, and subject these signatures to various trait levels and trait choice approaches. U. Rajendra Acharya et al. [4] have altruistically given shape to a novel technique for glaucoma recognition by means of a blend of quality and higher order spectra (HOS) traits from digital fundus images. Support vector machine, sequential minimal optimization, naïve Bayesian, and random-forest classifiers were employed to run the administered categorization. The promising outcomes attained by their method underlined the fact that the quality and HOS traits after z-score normalization and trait choice, when integrated with a random-forest classifier, was able to usher in superior quality of performance, pushing to the backstage the parallel classifiers. Their new-fangled traits were clinically momentous and were employed to identify glaucoma precisely.

M. Muthu Rama Krishnan et al. [5] have majestically conceived a minimal cost mechanized glaucoma identification mechanism by means of digital fundus images. Their work delved deeply into an appropriate system for the mechanized recognition of regular and glaucoma classes by means of Higher Order Spectra (HOS) and Discrete Wavelet Transform (DWT) traits. The mined traits were furnished to the Support Vector Machine (SVM) classifier with linear, polynomial order 1, 2, 3 and Radial Basis Function (RBF) to choose the finest kernel task for mechanized decision making. At last, they have come out with an innovative integrated index called Glaucoma Risk Index (GRI) which is composed of HOS and DWT traits, to identify the unidentified class by means of a solitary trait. Muthu Rama Krishnan and Oliver Faust [6] have jointly brought to light a minimal cost mechanized glaucoma identification method based on cross trait mining from digital fundus images. The paper threw light on a system for the mechanized recognition of regular and glaucoma classes by means of higher order spectra (HOS), trace transform (TT), and discrete wavelet transform (DWT) traits. The mined traits were furnished to a support vector machine (SVM) classifier with linear, polynomial order 1, 2,3 and radial basis function (RBF) with an eye on choosing the finest kernel for mechanized decision making. Moreover, they gave birth to an incorporated index called Glaucoma Risk Index (GRI) which was originated from HOS, TT, and DWT traits, to detect the unidentified class by means of a solitary trait.

\section{Proposed Glaucoma Detection Using Optimal Hyper Analytic Waveletco-Efficients}

Glaucoma is caused by increase of fluid pressure in the eye which damages the optic nerve and eventually causing blindness. Glaucoma detection technique from fundal retinal images using optimal hyper analytic wavelet coefficients is proposed in this paper. The optimal coefficients are found out using the hybrid GSO-Cuckoo search algorithm and would result in better classification of the Glaucoma. The proposed technique consists of four modules, namely pre-processing module, optimal transformation module, feature extraction module and finally classification module. The block diagram of the proposed technique is given in Fig. 1.

\subsection{Pre-processing module}

The sample size taken in this work is 158 images, out of 


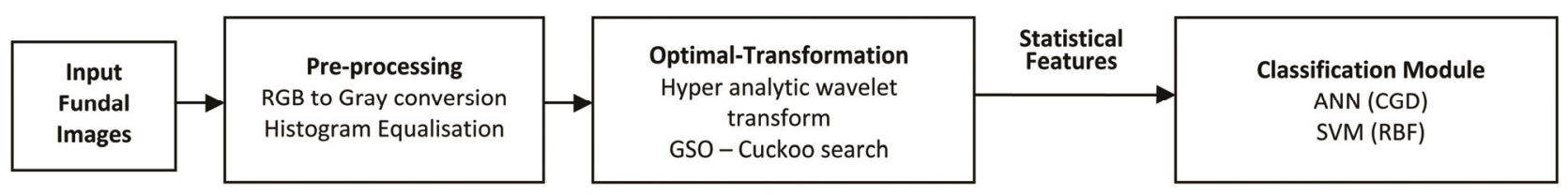

Fig. 1. The flowchart of the proposed technique

which 84 images belong to the normal class and 74 images belong to the glaucomatous class. We have executed 10fold cross validated test runs to ensure robustness of the classification.

The input fundus retinal images are preprocessed with the help of RGB to grey conversion and histogram equalization. The pre-processing makes the image fit for further processing by reducing the noise and enhancing the image.

\subsubsection{Grey scale conversion}

When the input image is in RGB format, it is converted to grey scale image. A grayscale digital image is an image in which the value of each and every pixel is a solitary model, in other words, it holds only intensity data. In a grayscale image, each and every pixel is a symbolized by the intensity value which fluctuates in the domain of 0 to 255. Therefore, grey scale images are absolutely composed of shades of grey, alternate from black at the lowest intensity with pixel value 0 to white at the highest with intensity value of 255 . Grayscale images are usually the consequence of calculating the intensity of light at each and every pixel in a solitary group of the electromagnetic spectrum.

\subsubsection{Histogram equalization}

Histogram equalization is used to improve the contrast of the image which would improve the feature extraction process. Histogram equalization is entrusted with the task of improving the contrast of the image by adapting the intensity of the image. Let the image to be equalized be represented by $I$ with a matrix dimension of $t a_{r} \times t a_{c}$ in which pixel intensities is in the range of 0 to $N a-1$. The number of probable intensity values is designated by $\mathrm{Na}$ which tends to be 256 as far as grey scale images are concerned. Let $h a_{m}$ symbolize the normalized histogram of $I$. The $h_{m}$ is furnished by the Eq. (1)

$$
\begin{gathered}
h_{m}=\frac{\text { number of pixels with intensity } \mathrm{m}}{\text { total number of pixels }}, \\
m=0,1, \ldots, N a-1
\end{gathered}
$$

The histogram equalized image $f a$ is givenby the Eq. (2)

$$
f a_{j, k}=\text { floor }(N a-1) \sum_{m=0}^{I_{j, k}} h_{m}
$$

\subsection{Optimal transformation module}

After pre-processing, the image is Hyper Analytic transformed from [15] which features would be extracted. The transformation is carried out after finding the optimal coefficients with the help of hybrid GSO cuckoo search optimization algorithm. The employment of optimal coefficients would result in having better classification of glaucoma giving good results.

Wavelets are mathematical functions that classify information into diverse frequency components. Wavelets are tasks that meet definite mathematical needs and are employed in symbolizing information or other similar tasks. The DWT (Discrete Wavelet Transform) is a type of wavelet which converts discrete signal from time domain into time frequency domain by means of the use of filter banks for the creation of the multi-resolution time-frequency plane. As regards the DWT, particular families of wavelet tasks are evolved like densely backed, orthogonal or bi-orthogonal and are symbolized by lowpass and high-pass scrutiny and synthesis filters. Both the approximate and detailed coefficient sub-bands hold significant information in statistical analysis [16]. The phase spectra in addition to the magnitude component significantly provide statistical information about the data (image) [17].

The wavelet coefficients $\mathrm{L}_{\mathrm{C}}$ and $\mathrm{H}_{\mathrm{C}}$ are modulated by the input angular parameters through the poly-phase representation method proposed by Vaidyanathan [22]. Their algorithm allows deriving orthonormal finite impulse response filters of chosen length.

The polyphase matrix proposed by vaidyanathan is given by Eq. (3)

$$
\begin{aligned}
H_{p}(Z) & =\left(\begin{array}{ll}
H_{00}(Z) & H_{01}(Z) \\
H_{10}(Z) & H_{11}(Z)
\end{array}\right) \\
& =\left(\begin{array}{cc}
C_{o} & S_{0} \\
-S_{0} & C_{o}
\end{array}\right) \prod_{i=1}^{N-1}\left(\begin{array}{cc}
1 & 0 \\
0 & Z^{-1}
\end{array}\right)\left(\begin{array}{cc}
C_{i} & S_{i} \\
-S_{i} & C_{i}
\end{array}\right)
\end{aligned}
$$

where the first and second columns correspond to even and odd taps of the filters; the first and second rows correspond to the low-pass and high-pass coefficients. $\mathrm{C}_{\mathrm{i}}$ and $\mathrm{S}_{\mathrm{i}}$ are the input angular parameters.

The Analytical Discrete Wavelet Transform (ADWT) is well-acknowledged as a complex wavelet transform, which does away with the employment of complex mother wavelets, and makes use of regular mother wavelets like 
the ones advocated by Daubechies even though it is essential that the transform is executed on the analytical signal linked with the input signal. The Hyper-analytic transform (HWT) is an annexe of the ADWT. The HWT of the image can be estimated by means of the 2D-DWT of its linked hyper complex image. This quality entails the legacy of any mother wavelet from 2D DWT for HWT. The HWT of an image $f(x, y)$ is given by: $H W T\{f(x, y)\}=\left\langle f(x, y), \psi_{a}(x, y)\right\rangle$, where $\psi_{a}$ is the hyper-complex mother wavelet associated to a real mother wavelet $\psi$. HWT uses four trees, each implemented by 2D-DWT giving rise to four set of coefficients given by $z+i, z-i, z+r$ and $z-r$. These would contain high pass and low pass filter coefficients. Let low pass filter coefficients be represented as $L_{C}=\left\{l_{1}, l_{2}, l_{3}, \ldots, l_{N}\right\}$. From these low pass coefficients, high pass coefficients are constructed which is represented by $H_{C}=\left\{h_{1}, h_{2}, h_{3}, \ldots, h_{N}\right\}$. The constructed high pass co-efficient is defined by the equation $h_{i}=(-1)^{i+1} l_{2 N-i}$. Both high pass and low pass coefficients would be optimized, indirectly from the angular parameters in (3), with the help of hybrid GSO cuckoo search optimization algorithm.

\subsection{GSO-Cuckoo search hybrid optimisation algorithm}

From times immemorial, it has been an enthusing phenomenon that the ever-mysterious nature has incessantly triggered the human being to continue with his insatiable thirst for treading into the hitherto unexplored horizons of investigation in the versatile domain of computation dipping him in delightful delirium. There is no second opinion regarding the fact that nature is the single most treasure house of charismatic concepts, pragmatic principles. The GSO [18] is a population-based optimization algorithm which exploits producer-scrounger model as a structure. Under this structure, conceptions and stratagems of resource hunting from animal probing attitude is followed symbolically. Standard animal scanning mechanisms are utilized for producers. Scrounging strategies of house are extensively applied in the GSO algorithm. In addition to the producers and scroungers, certain cluster members are scattered from a cluster to perform random walks to keep aloof entrapments in local minima. Cuckoo search (CS) [19] is one of the stylish optimization algorithms which has originated from the stimulus that the obligate brood parasitism of certain cuckoo range lays their eggs in the nests of different crowd birds owing allegiance to diverse species. In accordance with the required brood parasitic accomplishment of certain cuckoo range in combination with the Levy flight performance of certain birds and fruit flies, the algorithm is executed. In our paper, we hybridize GSO with cuckoo search algorithm [19] to have better results.

\subsubsection{GSO work-flow}

The population of the GSO algorithm is known by the name 'group' and each and every person in the group is called a member. As far as GSO is concerned, a group comprises three different kinds of members: producers and scroungers whose behaviour patterns are in harmony with the PS (Population Search) model in addition to dispersed members who are entrusted with the task of random walk motions. It is a foregone assumption that there cannot be more than one producer at each and hunting bout and the other members are scroungers and rangers. With the result, Group members are on the lookout for the patches. At every iteration, a group member, positioned in the utmost hopeful zone and offering the best fitness value, is selected as the producer. Thereafter, it waits and examines the environment to hunt for resources (optima). In GSO algorithm, fundamental scanning techniques launched by white crappie are exploited. The fitness function used is the accuracy in classification of Glaucoma. This is carried out with the help of feature extraction and neural networks. Feature extraction and classification is elaborated in section 3.3 and 3.4 respectively.

Let the input group having $N$ number of members be represented by $G P=[m 1, m 2, \ldots ., m N]$. Producers $P D$ (the best group members), scroungers $S C$ and dispersed members $D S$ can be roughly denoted as:

$$
\begin{gathered}
P D=[\text { mi }] \text { for } i=\text { members with best fitness } \\
S C=[\text { mj }], \text { for } j \neq \text { producer members } \\
D S=[\mathrm{mk}], \text { for } k \neq \text { scounger members }
\end{gathered}
$$

All members are measured to be in an n-dimensional search space having a pursuit distance $\lambda$ and pursuit angle $\varpi$. The $n$-dimensional space is defined by maximum pursuit angle $\varpi_{\max } \in R$ and maximum pursuit distance $\lambda_{\max } \in R$. Position of the producer is set as the apex. In the $k^{\text {th }}$ iteration, the producer $Y_{p}$ behaves as detailed below.

The producer initially scans at zero degree and then scans laterally by randomly sampling three points in the scanning field. One point at zero degree is defined by Eq. (4),

$$
Y_{z}=Y_{p}^{k}+n_{1} \cdot \lambda_{\max } U_{p}^{k}\left(\varpi^{k}\right)
$$

One point in the right hand side is defined by Eq. (5),

$$
Y_{r}=Y_{p}^{k}+n_{1} \cdot \lambda_{\max } U_{p}^{k}\left(\varpi^{k}+n_{2} \varpi_{\max } / 2\right)
$$

and one point in the left hand side is defined by Eq. (6),

$$
Y_{l}=Y_{p}^{k}+n_{1} \cdot \lambda_{\max } U_{p}^{k}\left(\varpi^{k}-n_{2} \varpi_{\max } / 2\right)
$$

where, $U$ is the directional unit vector, $n_{1}$ is a normally distributed random number with mean 0 and standard deviation 1 and $n_{2}$ is uniformly distributed random 
sequence in the range $[0,1]$.

As a result, producer attempts to locate the finest point with the greatest fitness value. If the finest point has a superior fitness value to its current position, then it would move to this point. Otherwise it remains in its current position and move its head to a fresh randomly generated angle furnished by Eq. (7),

$$
\varpi^{k+1}=\varpi^{k}+n_{2} \beta_{\max }
$$

where $\beta_{\max }$ is maximum turning angle.

In the mean time, if the producer is not able to locate a superior area after a set of iterations then it holds or maintains the value to zero degree ie. $\varpi^{k+1}=\varpi^{k}$.

In each and every probing iteration, a lot of group members are chosen as scroungers. The scroungers will keep hunting for occasions to come into contact with the resources located by the producer. At the $k^{\text {th }}$ iteration, the area replication attitude of the $j^{\text {th }}$ scrounger can be fashioned as a random walk in the direction of the producer. In the random walk of the $j^{\text {th }}$ scrounger, this is performed by supplementing an arbitrary locational value of the producers, which is easily gathered from the set of $Y$ values from the producers, as defined by Eq. (8),

$$
Y_{i}=Y_{i}^{k}+n_{3} \circ\left(Y_{p}^{k}-X_{j}^{k}\right)
$$

where, $n_{3}$ is a uniform random sequence in the range $(0$, 1). Operator "०" is the Hadamard product which computes the entry wise product of the two vectors. In the course of scrounging, the $j^{\text {th }}$ scrounger would go on hunting for similar occasion to come into contact with the producers. The remaining group members face the prospect of being dispersed from their current positions. If the $j^{\text {th }}$ group member is dispersed, it tends to accomplish ranging. The dispersed members are called 'rangers'. At the $k^{\text {th }}$ iteration, it produces a random head angle $\varpi_{i}$ and subsequently, selects an arbitrary distance furnished by: $\lambda=j \cdot n_{1} \lambda_{\max }$ and it travels to the fresh location furnished by:

$$
Y_{i}^{k+1}=Y_{i}^{k+1}+n_{1} \cdot \lambda_{i} D_{i}^{k}\left(\varpi^{k+1}\right)
$$

\subsubsection{Cuckoo search hybridization in the GSO work-flow:}

Hence, we get the updated values and in each iteration the worst cases (having worst fitness values) are replaced by the updated values using cuckoo search. The updation is carried out in each iteration of the GSO. Here the input to the cuckoo search would be worst cases obtained in GSO and each member represents an egg in the nest. These are evaluated using fitness function and updated using Levy flight to have new solutions. Let the host nests be symbolized by $H=\left\{h_{1}, h_{2}, \ldots, h_{N_{\text {cha }}}\right\}$.

Let the entity inside the host nest be represented as $Z_{i}=\left\{z_{i 1}, z_{i 2}, \ldots, z_{i N h}\right\}$, where $N h$ is the number of signals in the $i^{\text {th }}$ host nest. Then, Levi flight is carried out on $Z_{i}$ to yield to get a new cuckoo $Z_{i}^{*}$. Suppose the signal $z_{i 1}$ in $Z_{i}$, and then the updated value is given by:

$$
z_{i 1}{ }^{*}=z_{i 1}{ }^{(t+1)}=z_{i 1}{ }^{(t)}+\Delta \otimes \operatorname{Levy}(z)
$$

At this juncture, $\Delta$ is the step size which is a positive measure and is taken as one. The Levi flight equation implies the stochastic equation for arbitrary walk as it is dependent on the present position and the changeover possibility, furnished as second term in the equation. At this point, the levy sharing is specified by:

$$
\operatorname{Levy}(y)=\sqrt{\frac{c}{2 \pi}} \cdot \frac{e^{-\frac{1}{2}\left(\frac{c}{y}\right)}}{y^{3 / 2}}
$$

where, $c$ is arbitrary constant. Therefore, by executing Levi search, we get novel solutions and after that the fitness value (accuracy value) of the new solution is discovered. Let the fitness of the Levi achieved nest be $F t_{i}$.

Consequently, some other nest is observed as other than the $i^{\text {th }}$ host nest and let the nest in discussion be indicated by $Z_{j}=\left\{z_{j 1}, z_{j 2}, \ldots, z_{j N h}\right\}$ demonstrating $j^{\text {th }}$ host nest. The strength of the $j^{\text {th }}$ nest is demarcated by means of the fitness function and is represented by $F t_{j}$. If the fitness of the Levy flight made $i^{\text {th }}$ nest $F t_{i}$ superior than fitness of the $j^{\text {th }}$ nest $F t_{j}$, then substitute $j^{\text {th }}$ nest signal values $Z_{j}=\left\{z_{j 1}, z_{j 2}, \ldots, z_{j N g}\right\}$ by the $i^{\text {th }}$ host nest Levy performed values $Z_{i}{ }^{*}=\left\{z_{i 1}{ }^{*}, z_{i 2}{ }^{*}, \ldots, z_{i N g}{ }^{*}\right\}$. The sequence is completed for all the host nests $h_{i}$ where each of the nests are at first Levi flight performed, linked fitness is found out $F t_{i}$, compared to fitness of some other nest $F t_{j}$ and the substitution is performed if the condition $F t_{i}>F t_{j}$ is satisfied. In each and every iteration, a portion of the utmost horrifying nests are done away with

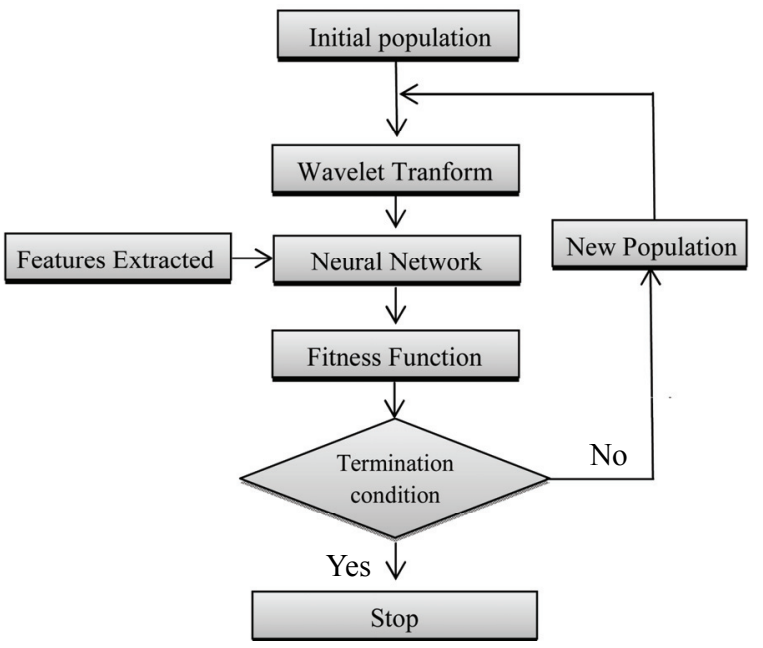

Fig. 2. The flow diagram of the optimal transformation module 
and fresh nests are constructed as replacement. Thus, we preserve the most outstanding remedies and replace the most horrible nests by freshly constructed nests. The full circle is continued until certain stop standard is fulfilled and the current top in the previous circle executed emerges as the top solution. This top solution replaces the worst members of the GSO algorithm. The flow diagram is given in Fig. 2.

\subsection{Feature extraction module}

After optimal transformation, the features are extracted from the images. We extract five features from the images including the mean, energy, entropy of magnitude, entropy of squared magnitude and entropy of cube magnitude.

By the term arithmetic mean, we generally mean the mean or average of the objects under deliberation and it is a technique to arrive at the central tendency of a sample space. Mean of the image is calculated by summing up all the pixel intensity values and then dividing it by the total number of pixels. The intensity of the pixels is $I_{j}$ for a total $\mathrm{N}_{\mathrm{p}}$ pixel. Here $\mathrm{Np}$ is the number of pixels in the image. The mean of the image $\mu$ can be given by Eq. (12),

$$
\mu=\frac{\sum_{j} I_{j}}{N p}
$$

\subsubsection{Energy}

Energy is used to describe a measure of information present in the image. It can be defined as the mean of squared intensity values of the pixels. The intensity of the pixels is $\mathrm{I}_{\mathrm{j}}$ for a total $\mathrm{N}_{\mathrm{p}}$ pixel. $\mathrm{Np}$ is the number of pixels in the image. The energy of the image $E$ can be given by Eq. (13),

$$
E=\frac{\sum_{j} I_{j}^{2}}{N p}
$$

\subsubsection{Entropy of magnitude}

Entropy $(e)$ is a measure of unpredictability or information content and is given by Eq. (14),

$$
e=-\sum_{i} P_{i} \log _{2} P_{i}
$$

In the above formula, $\mathrm{P}_{\mathrm{i}}$ is the probability that the difference between 2 adjacent pixels is equal to $\mathrm{i}$, and $\log _{2}$ is the base 2 logarithms.

\subsubsection{Entropy of squared magnitude}

Here, the entropy is found out after replacing the intensity value of pixel by the squared value. Suppose the intensity of image pixels be represented by $\operatorname{Iop}_{j}$ after the optimal transformation, where $\mathrm{o}<\mathrm{j} \leq \mathrm{Np}$ and $\mathrm{Np}$ is the number of pixels in the image. Then, entropy is found for image having pixel values of $\operatorname{Iop}_{\mathrm{j}}{ }^{2}$. After the modification in the intensity values, entropy is found using Eq. (15),

$$
e=-\sum_{i} P_{i} \log _{2} P_{i}
$$

In the above formula, $\mathrm{P}_{\mathrm{i}}$ is the probability that the difference between 2 adjacent pixels is equal to $i$, and $\log _{2}$ is the base 2 logarithms.

\subsubsection{Entropy of cube magnitude}

Here, the entropy is found out after replacing the intensity value of pixel by the cube value. Suppose the intensity of image pixels be represented by $\operatorname{Iop}_{j}$ after the optimal transformation, where $\mathrm{o}<\mathrm{j} \leq \mathrm{Np}$ and $\mathrm{Np}$ is the number of pixels in the image. Then, entropy is found for image having pixel values of $\operatorname{Iop}_{\mathrm{j}}{ }^{3}$. After the modification in the intensity values, entropy is found using Eq. (16),

$$
e=-\sum_{i} P_{i} \log _{2} P_{i}
$$

In the above formula, $\mathrm{P}_{\mathrm{i}}$ is the probability that the difference between 2 adjacent pixels is equal to $\mathrm{i}$, and $\log _{2}$ is the base 2 logarithms.

\subsection{Classification module}

\subsubsection{CGD- BPN-ANN}

After transforming the image with the optimal coefficients and extracting features from it, neural network is employed for classification. Artificial Neural Networks have emerged as an angel in times of need, extending a helping hand to the groping physicians' enabling them to evaluate, model and comprehend intricate medical information and images across a wide gamut of medical applications. In universal, the neural network comprises of three layers named as input layer, hidden layer and the output layer. The input of the neural network is the features extracted from image including the mean, energy and entropy of three types. The network is trained under a large set of different fundal retinal images in order to enable them to effectively detect presence of Glaucoma in a person in the testing phase. The neural network works making use of two phases, one is the training phase and the other is the testing phase. We have used feed forward neural network with back propagation algorithm.

\subsubsection{Training phase}

In the training phase, the input image is feature extracted 


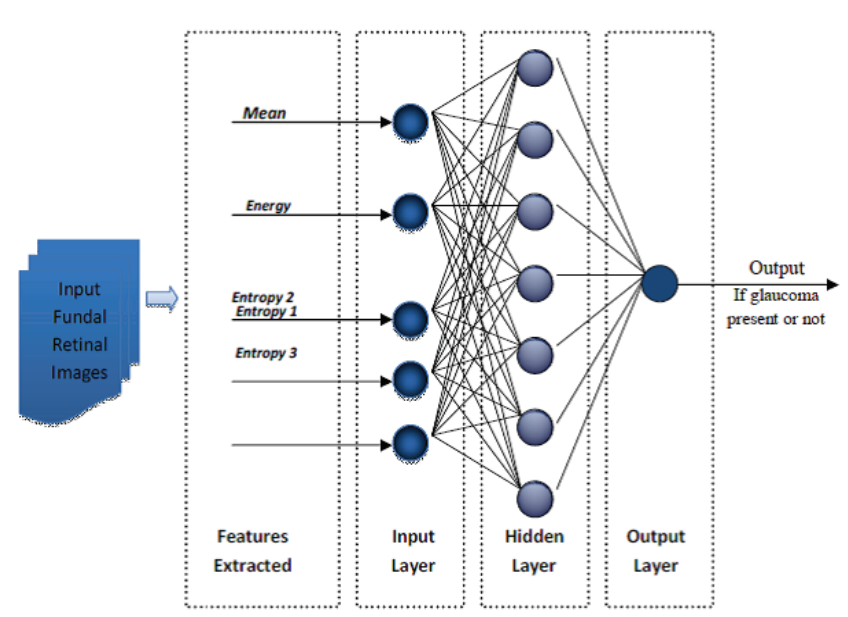

Fig. 3. Block diagram of the neural network

and this feature vector is given as the input to the neural network. At the outset, the nodes are offered random weights. As the output is, by now, identified in the training phase, the output acquired from the neural network is contrasted with the original and weights are altered by means of back-propagation algorithm with an eye on cutting down the inaccuracy. This task is performed for a substantial number of images targeted at arriving at a consistence mechanism with weights designated in the nodes. In this phase, fundal retinal images of both the normal person as well as person having glaucoma are given as input.

\subsubsection{Testing phase}

In the investigation stage, the input image is furnished to the trained neural network possessing specified weights in the nodes and the output is estimated so as to find if the person is having glaucoma or not. Fig. 3 shows the block diagram of the neural network.

\subsubsection{Support vector machines}

The problem is to categorize a set of " $n$ " training vectors with targets. Let $\mathrm{T}_{\mathrm{i}}$ be the training vectors and $\mathrm{Z}=[+1,-1]$ be the targets. In this example, the targets are diseased and normal images. The stages of the $\mathrm{T}$ which lie in the hyperplane should satisfy eqn W.T $+b=0$ where " $w$ " is the normal to the hyperplane, $|\mathrm{b}| /|| \mathrm{w}||$ is the perpendicular distance from the hyperplane to the origin. The condition is written as in equation $\mathrm{Z}_{\mathrm{i}}\left(\mathrm{W}^{\mathrm{T}} \mathrm{T}_{\mathrm{i}}+\mathrm{b}\right) \geq 1$. Hither, the kernel function chosen is a radial basis function by the equation, $k=\exp \left(\frac{\left\|T_{i}^{t}-T_{j}\right\|}{2 \sigma^{2}}\right)$, where $\sigma^{2}$ is the variance.

\section{Results and Discussion}

The results attained for the proposed glaucoma detection technique is given in this section. In section 4.1, the experimental set up and the evaluation metrics used are given. In section 4.2, simulation results are given and section 4.3 gives the performance analysis

\subsection{Experimental set up and evaluation metrics}

The proposed technique is implemented in MATLAB on a system having $6 \mathrm{~GB}$ RAM and $2.9 \mathrm{GHz}$ Intel i-7 processor. The database used for the evaluation is the RimOne database [20]. The database is composed of $169 \mathrm{ONH}$ images obtained from 169 full fundus images of different subjects. The ONH is a clearly identifiable part of the retina so these images have been manually cropped to this region of interest. The evaluation metrics used to evaluate the proposed technique are sensitivity, specificity and accuracy [21]. True positive, true negative, false negative and false positive are found out to find these metric values.

Table 1. Table defining the terms TP, FP, FN, TN

\begin{tabular}{c|c|c|c}
\hline $\begin{array}{c}\text { Experimental } \\
\text { outcome }\end{array}$ & $\begin{array}{c}\text { Condition as } \\
\text { determined by the } \\
\text { standard of truth }\end{array}$ & Definition & Representation \\
\hline Positive & Positive & $\begin{array}{c}\text { True Positive } \\
\text { (TP) }\end{array}$ & $\alpha$ \\
\hline Positive & Negative & $\begin{array}{c}\text { False Positive } \\
\text { (FP) }\end{array}$ & $\gamma$ \\
\hline Negative & Positive & $\begin{array}{c}\text { False Negative } \\
\text { (FN) }\end{array}$ & $\lambda$ \\
\hline Negative & Negative & $\begin{array}{c}\text { True Negative } \\
\text { (TN) }\end{array}$ & $\beta$ \\
\hline
\end{tabular}

Let true positive be represented as $\alpha$, true negative be represented as $\beta$, false positive be represented as $\gamma$ and false negative be represented as $\lambda$. Sensitivity $(\vartheta)$ is the proportion of true positives that are correctly identified by a diagnostic test. It shows how good the test is at detecting a disease. It is given by Eq. (17),

$$
\vartheta=\frac{\alpha}{\alpha+\lambda}
$$

Specificity $(\psi)$ is the proportion of the true negatives correctly identified by a diagnostic test. It suggests how good the test is at identifying normal (negative) condition, as defined by Eq. (18),

$$
\psi=\frac{\beta}{\beta+\gamma}
$$

Accuracy (represented by $\delta$ ) can be defined as the proportion of the true results ( $\alpha$ and $\beta$ ) in total results. Mathematically, it cane defined by Eq. (19),

$$
\delta=\frac{\alpha+\beta}{\alpha+\beta+\gamma+\lambda}
$$


Optimal Hyper Analytic Wavelet Transform for Glaucoma Detection in Fundal Retinal Images

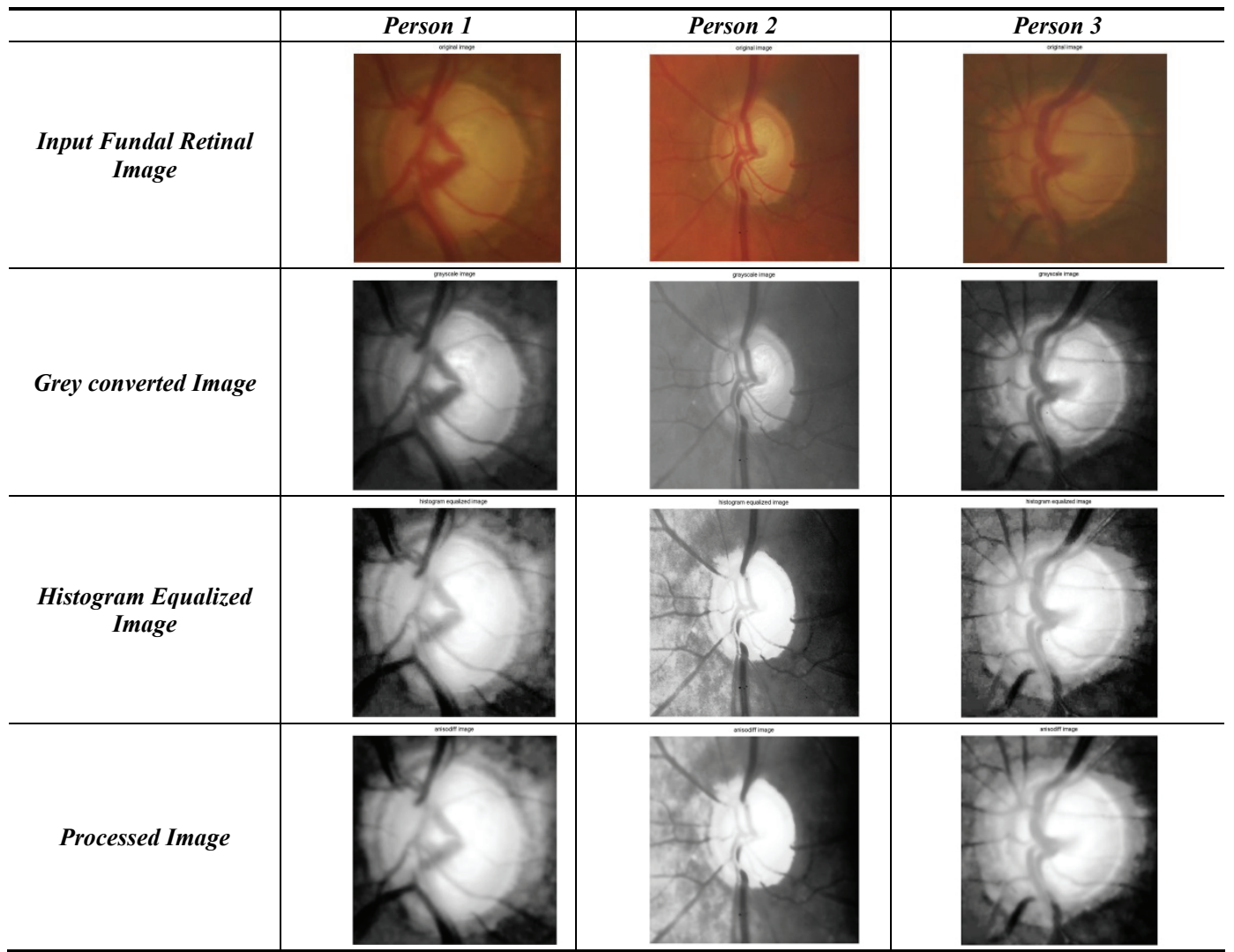

Fig. 4. Simulation experimental results

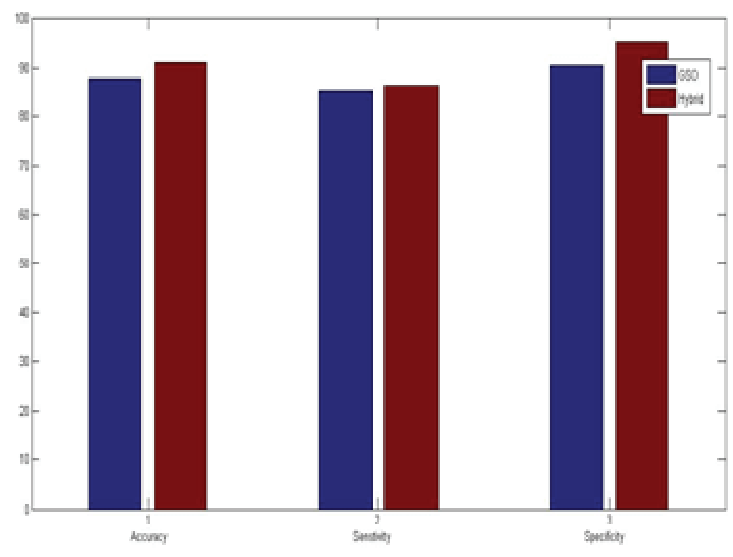

(a)

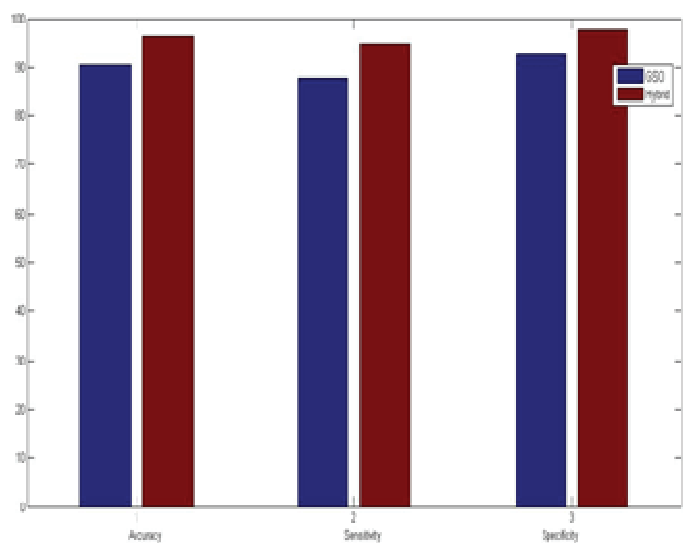

(b)

Fig. 5. Evaluation metrics for CGD-BPN ANN (a) and SVM (b) in test runs

\subsection{Simulation results}

The experimental simulation results obtained for the proposed technique are given in this section. The input image, grey converted image, histogram equalized image and processed images of three sample persons is given in Fig. 4.

\subsection{Performance analysis}

The proposed technique is evaluated in this section with
Table 2. Average evaluation metrics for GSO and Hybrid GSO

\begin{tabular}{c|c|c|c|c}
\hline & \multicolumn{2}{|c|}{$\begin{array}{c}\text { Test Run-I } \\
\text { ANN Classifier }\end{array}$} & \multicolumn{2}{c}{$\begin{array}{c}\text { Test Run-II } \\
\text { SVM Classifier }\end{array}$} \\
\hline & GSO & Hybrid & GSO & Hybrid \\
\hline Average Accuracy & $87.97 \%$ & $91.13 \%$ & $90.5 \%$ & $96.2 \%$ \\
\hline Average Sensitivity & $85.13 \%$ & $86.49 \%$ & $87.84 \%$ & $94.59 \%$ \\
\hline Average Specificity & $90.47 \%$ & $95.24 \%$ & $92.86 \%$ & $97.62 \%$ \\
\hline
\end{tabular}

the use of evaluation metrics of accuracy, sensitivity and specificity. The performance is compared with the GSO 
and the hybrid framework. The execution is done in two test runs with the classifiers, CGD-BPN ANN and SVM. Fig. 5 shows the metrics evaluated (for GSO and the hybrid) with ANN and SVM classifiers, correspondingly.

\subsection{Optimized wavelet coefficients:}

From the Fig. (5), we can infer that the highest performance is attained by the SVM classifier in the hybrid GSO

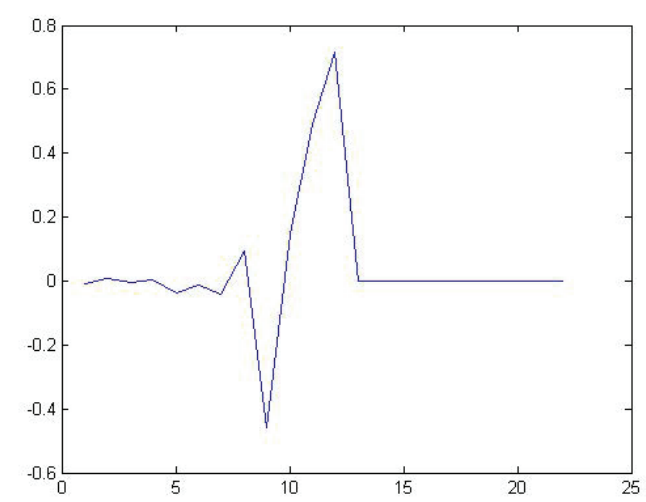

(a)

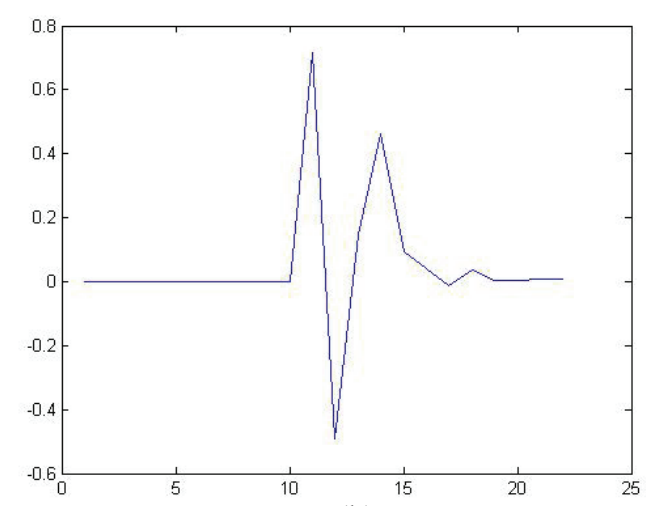

(b)

Fig. 6. Optimized Scaling function (a) and optimized wavelet function (b).

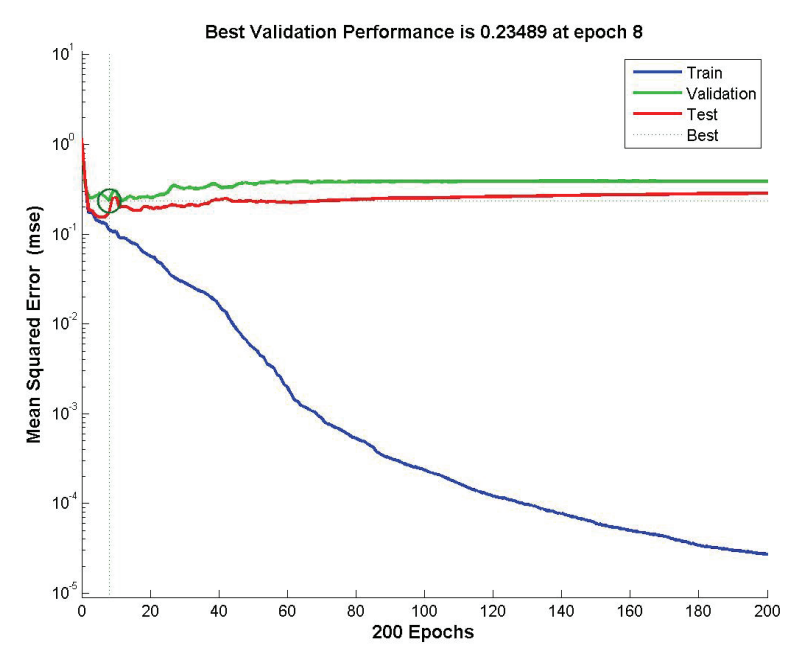

Fio. 7. Validation nerformance chart mode. In detail, the angular parameters (here length is 11) are directly optimized by the hybrid GSO, then the wavelet transform parameters (22-tap low pass and high pass) are generated from the angular parameters. Fig. 6 show the optimized low-pass and high-pass coefficients that attain the highest fitness with SVM.

Fig. 7 gives the validation performance chart for 200 epochs with the optimized scaling and wavelet function from the test-run-I. Best validation performance is obtained was 0.23489 for epoch 8 .

\subsection{Discussion}

The proposed hybrid GSO has outperformed the conventional GSO with 4 and $6 \%$ raise in accuracy with the ANN and SVM classifiers respectively. The increase in sensitivity is 1 and $7 \%$ with the ANN and SVM classifiers respectively.

The increase in specificity is $5 \%$ for both the classifiers. Regarding the classifiers SVM consistently out-performed the ANN.

In [22], the glaucoma detection accuracy was high to a level of $81 \%$ by incorporating the magnitude and phase information through complex wavelet transform. However, the non-specificity of the mother wavelets for the corresponding application was not accounted. The proposed work establishes the significance of the application specific wavelets with an average accuracy of $85 \%$.An example for application-specific mother wavelet design was proposed in [23] where the wavelet coefficients were optimized for ECG beat classification. The customization was done through Particle Swarm Optimization and the accuracy was around $75 \%$. Our novel proposal to hybridize Cuckoo search philosophy, to destroy the worst solutions, in the GSO framework evolved with a raise in accuracy over GSO for the same data-set in the 2 test runs.

\section{Conclusion}

Glaucoma detection technique from fundal retinal images using optimal hyper analytic wavelet coefficients is proposed in this paper. The optimal coefficients are found out using the hybrid GSO-Cuckoo search algorithm. The proposed hybrid technique has given high accuracy, sensitivity and specificity values than the conventional GSO. In the hybrid population, the search space is maintained large as new (distant) members are laid-in by the Cuckoos. This prevents the fitness from prematurely trapped and discloses more fit solutions.

\section{References}

[1] Jun Cheng, Jiang Liu, Yanwu Xu and Fengshou Yin, 
"Superpixel Classification Based Optic Disc and Optic Cup Segmentation for Glaucoma Screening", IEEE transactions on Medical Imaging, vol. 32, no. 6, pp. 1019-1032, 2013.

[2] Kim P.Y, Iftekharuddin K.M, Davey P.G. and Toth. M, "Novel Fractal Feature-Based Multiclass Glaucoma Detectionand Progression Prediction", IEEE Journal of Biomedical and Health Informatics, vol. 17, no. 2, pp. 269 - 276, 2013.

[3] Dua. S, Acharya. U. R, Chowriappa. P and Sree. S.V, "Wavelet-Based Energy Features for Glaucomatous Image classification", IEEE Transactions on Information Technology in Biomedicine, vol. 16, no. 1, pp. 80 - 87, 2012.

[4] Rajendra Acharya, Dua.S, Xian Du and Vinitha Sree. S, "Automated Diagnosis of Glaucoma Using Texture and Higher Order Spectra Features", IEEE Transactions on Information Technology in Biomedicine, vol. 15, no. 3, pp. 449 - 455, 2011.

[5] Muthu Rama Krishnan. M, Rajendra Acharya. U, Lim Choo Mina, Andrea Petznick, Jasjit S. Suri, "Data mining technique for automated diagnosis of glaucoma using higher order spectra and wavelet energy features", Knowledge-Based Systems, vol. 33, pp. 73-82, 2012.

[6] Muthu Rama Krishnan. M And Oliver Faust, J "Automated Glaucoma Detection Using Hybrid Feature Extraction In Retinal Fundus Images." Mech. Med. Biol, vol. 13, no. 1, pp. 1350011-1-21, 2012.

[7] Patton. N, Aslam. T.M, et al., "Retinal image analysis: Concepts, applications and potential", Progress in Retinal and Eye Research, vol. 25, no. 1, pp. 99-127, 2006.

[8] Anderson. D. R, "The Optic Nerve in Glaucoma" Duane's Ophthalmology, chapter 48, 2009.

[9] Chrastek. R, Wolf. M, et al, "Automated segmentation of the optic nerve head for diagnosis of glaucoma", Journal of Medical Image Analysis in Elsevier, Functional Imaging and Modeling of the Heart", vol. 9, no. 4, pp. 297-314, 2005.

[10] Ravishankar. S, Jain. A, Mittal. A, "Automated feature extraction for early detection of diabetic retinopathy in fundus images", in proceedings of IEEE con-ference on Computer Vision and Pattern Recognition, Miami, FL, pp. 210-217, 2009.

[11] K. Narasimhan, K. Vijayarekha, "An Efficient Automated System For Glaucoma Detection Using Fundus Image", Journal of Theoretical and Applied Information Technology", vol. 33, no.1, pp. 104-110, 2011.

[12] Rüdiger Bock, Jörg Meier, László G. Nyúl, Georg Michelson, Joachim Hornegge, "Retina Image Analysis System for Glaucoma Detection", in proceedings of German Society for Biomedical Engineering, pp.2629, 2007.

[13] K. R. Sung, Jong. S. Kim, et al., "Imaging of the retinal nerve fiber layer with spectral domain optical coherence tomography forglaucoma diagnosis," Br. J. Ophthalmol., vol. 95, no. 7, pp. 909-914, 2010.

[14] B. Brown, "Structural and functional imaging of the retina: New ways to diagnose and assess retinal disease," Clin. Exp. Optometry, vol. 91, no. 6, pp. 504-514, 2008.

[15] Ioana Adam, Corina Nafornita, Jean-Marc Boucher, AlexandruIsar, "A Bayesian Approach of Hyperanalytic Wavelet Transform Based Denoising", in proceedings of IEEE International Symposium on Intelligent Signal Processing, 2007, pp. 1 - 6, 2007.

[16] C. Raja and N. Gangatharan, "Appropriate sub-band selection in wavelet packet decomposition for automated glaucoma diagnosis." International Journal of Automation and computing, 2015. DOI 10.1007/ s11633-014-0858-6.

[17] C. Raja and N. Gangatharan, "Incorporating Phase Information for efficient Glaucoma Diagnosis through Hyper Analytic Wavelet Transform", in Proceedings of Fourth International Conference on Soft Computing for Problem Solving, Advances in Intelligent Systems and Computing, Vol. 2, pp. 325-339.

[18] S. He, Q. H. Wu, and J. R. Saunders, "Group Search Optimizer: An Optimization Algorithm Inspired by Animal Searching Behavior", in IEEE transactions on evolutionary computation, vol. 13, no. 5, pp. 973990, 2009.

[19] Xin She Yang and Deb, Suash, "Cuckoo search via Lévy flights", in IEEE proceedings World Congress on Nature \& Biologically Inspired Computing, pp. 210-214, 2009.

[20] Fumero.F, Alayon.S, et al., "RIM-ONE: An open retinal image database for optic nerve evaluation", in proceedings of International Symposium on ComputerBased Medical Systems, pp. 1-6, 2011.

[21] Wen Zhu, Nancy Zeng, Ning Wang, "Sensitivity, Specificity, Accuracy, Associated Confidence Interval and ROC Analysis with Practical SAS Implementations", in proceedings of the SAS Conference, Baltimore, Maryland, pages: 9, 2010.

[22] C.Raja and N.Gangatharan, "Glaucoma Detection in Fundal Retinal Images Using Trispectrum and Complex Wavelet-Based Features", European Journal of Scientific Research, vol. 97, no. 1, pp. 159-171, 2013.

[23] Abdelhamid Daamouche, Latifa Hamami, et al, "A wavelet optimization approach for ECG signal classification." Biomedical Signal Processing and Control, vol.7, pp. 342- 349, 2012.

[22] P.P. Vaidyanathan, "Multirate Systems and Filter Banks”, Prentice-Hall, Englewood Cliffs, 1993. 


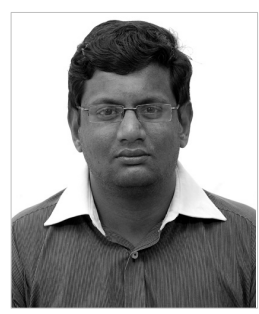

C. Raja received the B.E. Degree in Electronics and Communication Engineering in 2003 from Bharathidasan University and the M.Tech. Degree in Biomedical Signal Processing and Instrumentation in 2005 from SASTRA University, India. $\mathrm{He}$ is currently pursuing Ph.D. degree from Anna University, Chennai. Since 2005 he has been working as Assistant Professor in various Engineering colleges in India. Since May 2013, he has been working as Assistant Professor, Department of ECE, Anjalai Ammal Mahalingam Engineering College, India. To his credit he has published 5 research papers in international journals and international level conference proceedings. His research interests concern digital image processing, wavelets and swarm intelligence.

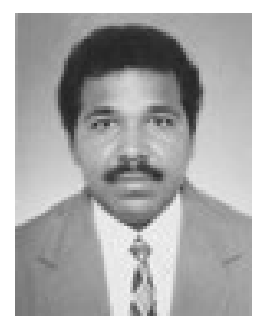

N. Gangatharan received the B.E. Degree in Electronics and Communication Engineering in 1988 and the M.E. Degree in Microwave and Optical engineering in 1990, both from Madurai Kamaraj University, India. $\mathrm{He}$ is a Second Ranker in the M.E. Degree Examinations of Madurai Kamaraj University in 1990. He received his second M.E. Degree in Computer Science and Engineering from Manonmaniam Sundaranar University, Tirunelveli, India, in 1997, and his M.B.A. Degree in Finance and Marketing from Madurai Kamaraj University, India, in 1999. He was on the Faculty of Electronics and Communication Engineering at the Indian Engineering College, Vadakkangulam, India, as a Professor from 1997 to 2001. From 2001 to 2005, he was on the Faculty of Engineering, Multimedia University (MMU), Cyberjaya Campus in Malaysia. From 2005 to 2006, he was on the Faculty of Electrical Engineering, Universiti Teknologi MARA (UiTM), Shah Alam in Malaysia. He took his Ph.D. degree in Telecommunication Engineering from Multimedia University in Malaysia. From January 2007 to January 2009, he was on the Faculty of Engineering at the Kigali Institute of Science and Technology (KIST), Kigali, Rwanda. From February 2009 to September 2010, he was an ICT Consultant at Rwanda Utilities Regulatory Agency (RURA), Rwanda. Since September 2010, he has been working as Professor \& Head, Department of ECE, RMK College of Engineering \& Technology, Chennai. To his credit he has published more than 25 research papers in international journals and conference proceedings. His research interests concern two dimensional and multidimensional digital signal processing, image processing, multimedia coding and telecommunication. 